\title{
Transgenic mitochondrial superoxide dismutase and mitochondrially targeted catalase prevent antiretroviral- induced oxidative stress and cardiomyopathy
}

James J Kohler', Ioan Cucoranu', Earl Fields, Elgin Green, Stanley He, Amy Hoying, Rodney Russ, Allison Abuin, David Johnson, Seyed H Hosseini, C Michael Raper and William Lewis

Transgenic mice (TG) were used to define mitochondrial oxidative stress and cardiomyopathy (CM) induced by zidovudine (AZT), an antiretroviral used to treat HIV/AIDS. Genetically engineered mice either depleted or overexpressed mitochondrial superoxide dismutase (SOD2 ${ }^{+/}$KOs and SOD2-OX, respectively) or expressed mitochondrially targeted catalase (mCAT). TGs and wild-type (WT) littermates were treated (oral AZT, 35 days). Cardiac mitochondrial $\mathrm{H}_{2} \mathrm{O}_{2}$, aconitase activity, histology and ultrastructure were analyzed. Left ventricle (LV) mass and LV end-diastolic dimension were determined echocardiographically. AZT induced cardiac oxidative stress and LV dysfunction in WTs. Cardiac mitochondrial $\mathrm{H}_{2} \mathrm{O}_{2}$ increased and aconitase was inactivated in $\mathrm{SOD} 2^{+/-} \mathrm{KOs}$, and cardiac dysfunction was worsened by AZT. Conversely, the cardiac function in SOD2-OX and mCAT hearts was protected. In SOD2-OX and mCAT TG hearts, mitochondrial $\mathrm{H}_{2} \mathrm{O}_{2}$, LV mass and LV cavity volume resembled corresponding values from vehicle-treated WTs. AZT worsens cardiac dysfunction and increases mitochondrial $\mathrm{H}_{2} \mathrm{O}_{2}$ in SOD2 ${ }^{+/-} \mathrm{KO}$. Conversely, both SOD2-OX and mCAT TGs prevent or attenuate AZT-induced cardiac oxidative stress and LV dysfunction. As dysfunctional changes are ameliorated by decreasing and worsened by increasing $\mathrm{H}_{2} \mathrm{O}_{2}$ abundance, oxidative stress from $\mathrm{H}_{2} \mathrm{O}_{2}$ is crucial pathogenetically in AZT-induced mitochondrial CM.

Laboratory Investigation (2009) 89, 782-790; doi:10.1038/labinvest.2009.39; published online 27 April 2009

KEYWORDS: mitochondria; cardiomyopathy; oxidative stress; antiretroviral; toxicity

Zidovudine ( $3^{\prime}$-azidothymidine, AZT) is an important antiretroviral nucleoside analog (NRTI) used to treat HIV/AIDS, ${ }^{1}$ but mitochondrial side effects, including cardiomyopathy $(\mathrm{CM}){ }^{2}$ limit the usefulness of AZT and its congeners. ${ }^{3-7}$ To elucidate mitochondrial mechanisms of AZT CM, transgenic mice (TG) served as experimental tools in vivo.

Mice with transgenic manipulation of manganesecontaining superoxide dismutase (SOD2 or MnSOD) and catalase (CAT) were employed to define AZT-induced mitochondrial oxidative stress. SOD2 is a mitochondrial enzyme that catalyzes dismutation of $\mathrm{O}_{2}^{-}$into $\mathrm{H}_{2} \mathrm{O}_{2}+\mathrm{O}_{2}$, but $\mathrm{H}_{2} \mathrm{O}_{2}$ itself also produces oxidative injury. ${ }^{8,9}$ Mitochondria eliminate $\mathrm{H}_{2} \mathrm{O}_{2}$ primarily through the enzyme activity of glutathione peroxidase, ${ }^{10}$ while oxidizing glutathione (GSH) to GSSG or through peroxiredoxins. ${ }^{11-13}$ These enzymes prevent $\mathrm{H}_{2} \mathrm{O}_{2}$ accumulation, limit formation of reactive species (eg, the hydroxyl radical, from $\mathrm{H}_{2} \mathrm{O}_{2}$ reactions with reduced iron or copper ${ }^{14}$ ), prevent left ventricular remodeling $^{11}$ and cardiotoxicity. ${ }^{15}$ In peroxisomes, catalase is an enzyme that decomposes $\mathrm{H}_{2} \mathrm{O}_{2}$ from flavoprotein reactions. ${ }^{16}$ Mitochondria use glutathione peroxidase to carry out a similar function. Genetically engineered mice that express catalase in mitochondria show decreased susceptibility to various forms of oxidative stress ${ }^{17,18}$ and were considered a model that may show resistance to oxidative effects of AZT.

In ' 2 by 2 ' factorial protocols, SOD2 $2^{+/-}$KOs exhibited reduced myocardial antioxidant defenses ${ }^{19}$ ('loss of function') that was worsened by AZT. SOD2-overexpressor TGs (SOD2OX) and mCAT (mitochondrially targeted catalase) TGs were each protected against AZT-induced cardiac oxidative stress $^{5,20}$ ('gain of function'). Data indicate that AZT CM in the heart involves $\mathrm{H}_{2} \mathrm{O}_{2}$ production.

Department of Pathology, Emory University, Atlanta, GA, USA

Correspondence: Dr W Lewis, MD, Department of Pathology, Emory University School of Medicine, 7117 Woodruff Memorial Building, 101 Woodruff Circle, Atlanta, GA 30322, USA.

E-mail: wlewis@emory.edu

${ }^{1}$ These authors contributed equally to this work.

Received 3 February 2009; revised 14 March 2009; accepted 24 March 2009 


\section{MATERIALS AND METHODS Mice and Genotyping}

TG mice were obtained from primary investigators' laboratories. SOD2 ${ }^{+/-} \mathrm{KOs}^{19}$ were from Brian Day and colleagues at the National Jewish Medical Research Center, Denver, CO, USA. SOD2-OX TG were from Ye-Shih Ho et al ${ }^{21}$ at Wayne State University, Detroit, MI, USA. The mCAT TGs ${ }^{22}$ were from Peter Rabinovitch and colleagues at the University of Washington, Seattle, WA, USA. TGs were either developed on a background of or bred congenically to C57BL/6.

\section{AZT Treatment Protocols}

All procedures complied with Emory Institutional Animal Care and Use Committee and NIH guidelines. AZT was from the manufacturer. Wild-type (WT) and TG littermates (both genders) were age-matched (8-12 weeks) at the start of AZT or vehicle treatment. Rodent chow and water were provided ad libitum in a $12 \mathrm{~h}$ light:dark, humidity and temperature controlled environment at Emory. AZT was administered daily by gavage in doses that resemble those used in human therapy. Mice received vehicle control ( $1 \%$ carboxymethylcellulose) or vehicle containing AZT $(0.22 \mathrm{mg}$ per day; $0.25 \mathrm{ml}$ ). At day 35 , echocardiographic measurements were made, animals killed, heart samples retrieved, and immediately processed for mitochondrial $\mathrm{H}_{2} \mathrm{O}_{2}$ quantification and aconitase activity, or were fixed for histology and electron microscopy (EM).

\section{Echocardiography of TG Mice}

Mice were anesthetized with Avertin $(0.25 \mathrm{mg} / \mathrm{g})$ and twodimensionally targeted $\mathrm{M}$-mode images were taken from the short axis (at the level of the largest LV diameter) using a $15 \mathrm{MHz}$ transducer (Acuson Sequoia Siemens USA, New York, NY, USA). In a blinded fashion, M-mode measurements of heart rate, left ventricle end-diastolic dimensions (LVEDD), left ventricle end-systolic dimensions, anterior wall thickness (AWTH) and posterior wall thickness (PWTH) were obtained from original tracings by using the leadingedge convention of the American Society of Echocardiography and by using the steepest continuous endocardial echoes. Left ventricle mass (LV mass) was computed using the formula: $\quad \mathrm{LV}$ mass $=1.05 \times\left((\mathrm{LVEDD}+\mathrm{AWTH}+\mathrm{PWTH})^{3}\right.$ $\left.-(\text { LVEDD })^{3}\right)$. LV mass for each mouse was normalized using its body weight $(\mathrm{mg} / \mathrm{g})$.

\section{Mitochondria Isolation}

Heart mitochondria were isolated fresh using the MITOISO1 kit (Sigma-Aldrich, St Louis, MO, USA). Approximately $10 \mathrm{mg}$ (wet weight) of freshly isolated myocardial tissue was put into buffer A (10 mM HEPES, pH 7.5, containing $0.2 \mathrm{M}$ mannitol, $70 \mathrm{mM}$ sucrose and $1 \mathrm{mM}$ EGTA) at $4^{\circ} \mathrm{C}$, and pretreated with $0.25 \mathrm{mg} / \mathrm{ml}$ trypsin and homogenized using a manual glass:glass homogenizer. Homogenization was followed by serial low $(1000 \mathrm{~g})$, and high-speed $(8500 \mathrm{~g})$ centrifugations. The resultant pellet was resuspended and stored in $10 \mathrm{mM}$ HEPES solution, $\mathrm{pH} 7.4$ containing $250 \mathrm{mM}$ sucrose, $1 \mathrm{mM}$ ATP, $0.08 \mathrm{mM}$ ADP, $5 \mathrm{mM}$ sodium succinate, $2 \mathrm{mM} \mathrm{K}_{2} \mathrm{HPO}_{4}$ and $1 \mathrm{mM}$ DTT.

\section{Mitochondrial $\mathrm{H}_{2} \mathrm{O}_{2}$ Abundance}

To quantitate mitochondrial $\mathrm{H}_{2} \mathrm{O}_{2}$, the Amplex Red assay kit (Molecular Probes, Eugene, OR, USA) was employed. This kit sensitively measures $\mathrm{H}_{2} \mathrm{O}_{2}$ by the horseradish peroxidasecatalyzed oxidation of colorless and non-fluorescent $\mathrm{N}$-acetyl-3, 7-dihydroxyphenoxazine that is oxidized to resorufin. When excited at $530 \mathrm{~nm}$, it emits light at $590 \mathrm{~nm}$ and this is stoichiometrically quantitated.

Fresh mitochondrial extracts isolated from cardiac myocytes (described above using MITOISO1 kit) were added to Amplex Red reagent $(10 \mu \mathrm{mol} / \mathrm{l})$ and horseradish peroxidase $(0.2 \mathrm{U} / \mathrm{ml})$ for $60 \mathrm{~min}$ at $37^{\circ} \mathrm{C}$ in $1 \times$ reaction buffer, protected from light. Fluorescence was detected at $590 \mathrm{~nm}$ using an excitation of $530 \mathrm{~nm}$ in a microtiter plate reader. Background fluorescence was subtracted. $\mathrm{H}_{2} \mathrm{O}_{2}$ release was calculated using $\mathrm{H}_{2} \mathrm{O}_{2}$ standards and expressed in nmol per mg protein per minute.

\section{Aconitase Activity}

Aconitase inactivation is a sensitive indicator of oxidative stress. $^{23,24}$ To assay aconitase, fresh myocardium $(10 \mathrm{mg}$ wet weight) was homogenized in ice-cold $50 \mathrm{mM}$ Tris- $\mathrm{HCl}(\mathrm{pH}$ 7.4) containing $0.6 \mathrm{mM} \mathrm{MnCl}_{2}, 1 \mathrm{mM}$ cysteine, $1 \mathrm{mM}$ citrate and $0.5 \%$ Triton-X 100. Enzyme activity was measured spectrophotometrically by monitoring the formation of cis-aconitate from isocitrate (per $\mathrm{mg}$ protein) as described. ${ }^{24}$

\section{Histological Analyses}

Heart samples were processed, sectioned $(6 \mu \mathrm{m})$ and stained with hematoxylin and eosin (H\&E). Slides were examined microscopically on a Nikon 800 Ultraphot Microscope (Nikon, Melville, NY, USA). Microscopic images were viewed by two observers in a blinded fashion and compared histopathologically. A semiquantitative scale was used for histopathological comparisons within the experiments.

Myocytolysis served as an indicator of cell damage. Semiquantitative analysis was carried out on microscopic sections (above) and reviewed independently by two investigators (WL and JJK). Total lytic myocytes were counted in each high-power field. Incidence of myocytolysis were determined using the following schema: zero myocytes (scored as a grade zero); 1-3 myocytes (scored as grade 1); 4-6 myocytes (scored as grade 2); 7-9 myocytes (was scored as grade 3); 10 or more myocytes (scored as grade 4). Averages from five high-power field images were taken from each slide and numerically scored according to the schema. On breaking the code, numerical data obtained from this scoring system were statistically analyzed. 


\section{Ultrastructural Pathological Evaluations (Electro Microscopy) of Cardiac Mitochondria}

Samples from KOs, TGs and WT murine hearts were evaluated by EM to define mitochondrial fine structure changes. Myocardial samples (approximately $1 \mathrm{~mm}$ cubes) were cut, rapidly fixed in diluted Karnovsky's fixative, and processed. $^{2,25,26}$ Thin sections $(0.5 \mu)$ were cut with a glass knife and stained with toluidine blue for orientation. Ultra thin (900 $\AA$ ) sections were cut with a diamond knife, stained with uranyl acetate and lead citrate and viewed on a Philips Morgagni electron microscope (Philips, Amsterdam, The Netherlands). Each EM image was reviewed by two investigators for structural abnormalities (eg, intramitochondrial lamellar bodies, cristae reduplication). ${ }^{2,25,26}$

\section{Experimental Analysis and Statistics}

Values for LV Mass, LVEDD, mitochondrial $\mathrm{H}_{2} \mathrm{O}_{2}$ and aconitase were compared in WT, TGs and AZT-treated cohorts using ANOVA in GraphPad Prism 4 (GraphPad, San Diego, CA, USA). Post-hoc testing used Newman-Keul's and unpaired $t$-test. A value of $P<0.05$ (determined by student's unpaired $t$-test) was considered statistically significant.

\section{RESULTS}

\section{General}

During the experimental protocols, no morbidity or mortality occurred in any cohort and final body weights were comparable (Figure 1).

\section{Final Body Weights (after 5wks treatment)}

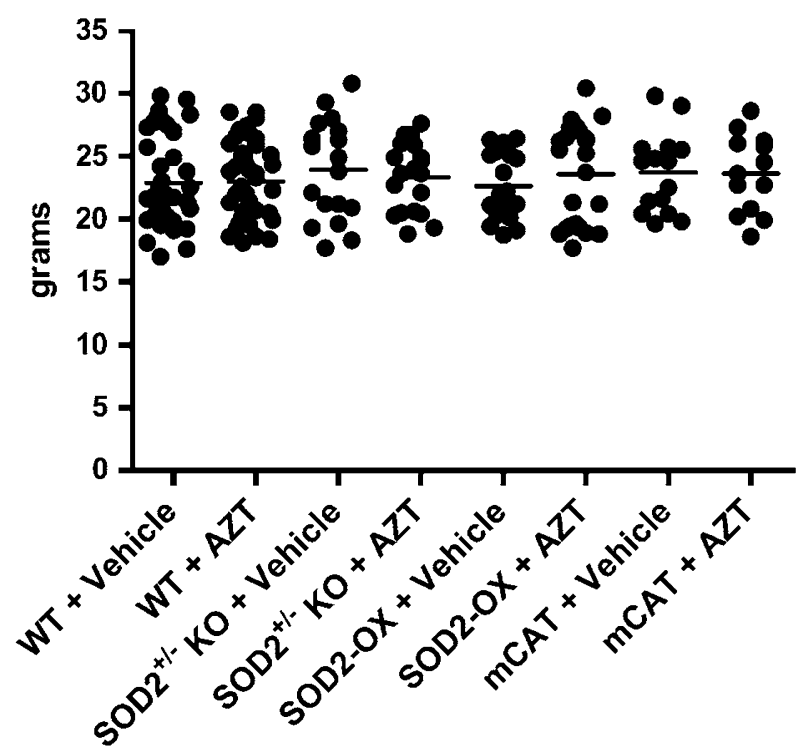

Figure 1 Final body weight after 5 weeks AZT treatment. Final body weights after 5 weeks treatment with vehicle and/or AZT were measured for individual mice and grouped by cohorts. All cohorts exhibited equivalent final body weights.

\section{Phenotype in SOD2 ${ }^{+/-}$KOs Compared With SOD2-OX or MCAT TGs}

Left ventricular mass and LVEDD were derived from direct echocardiographic measurements in each mouse to define the effects of AZT treatment, transgenesis or both on LV. AZT increased LV mass in WTs $(P<0.001)$ compared with that of vehicle-treated WTs (Figure $2 \mathrm{a})$. In vehicle-treated SOD2 ${ }^{+/-}$ KOs, LV mass increased $(P<0.001)$ (Figure 2a), however, AZT treatment of SOD2 ${ }^{+/-}$KOs further increased the LV mass (Figure 2a) compared with all other cohorts $(P<0.001)$. This observation suggested at least an additive effect of AZT on $\mathrm{LV}$ mass occurred in SOD2 ${ }^{+/-}$KOs. In contrast, LV mass in SOD2-OX and mCAT TGs (with or without AZT treatment) remained unchanged from that of vehicle-treated WTs (Figure 2a). In this latter scenario, overexpression of SOD2 or mCAT in TGs protected them against the AZT deleterious effects seen in SOD2 ${ }^{+/-}$KOs.

In contrast to LV mass, LVEDD in WTs was unchanged by AZT treatment (Figure 2b) and remained unchanged in vehicle-treated SOD2 ${ }^{+/-} \mathrm{KOs}$, in vehicle and AZT-treated SOD2-OX, and in vehicle and AZT-treated mCAT TGs (Figure 2b). However, LVEDD increased significantly in SOD $2^{+1-}$ KOs treated with AZT (Figure $2 \mathrm{~b}$ ), indicating that LV dilation in addition to LV hypertrophy (and thus bona fide $\mathrm{CM}$ ) resulted from AZT treatment in this cohort.

\section{Cardiac Mitochondrial $\mathrm{H}_{2} \mathrm{O}_{2}$ Abundance}

Cardiac mitochondrial $\mathrm{H}_{2} \mathrm{O}_{2}$ abundance was a quantitative index for oxidative events from AZT treatment, transgenesis or both. $\mathrm{H}_{2} \mathrm{O}_{2}$ in isolated mitochondria from heart samples from $\mathrm{SOD} 2^{+1-} \mathrm{KOs}$ was increased with AZT treatment (Figure 3a) compared with $\mathrm{H}_{2} \mathrm{O}_{2}$ abundance in cardiac mitochondrial samples from vehicle-treated $\mathrm{SOD} 2^{+/-} \mathrm{KOs}$ or WTs (with or without AZT). $\mathrm{H}_{2} \mathrm{O}_{2}$ abundance in AZTtreated SOD2-OX cardiac mitochondria was comparable with that of vehicle-treated WT cardiac mitochondria (Figure 3a). In vehicle-treated mCAT TGs, cardiac mitochondrial $\mathrm{H}_{2} \mathrm{O}_{2}$ abundance seemed lower, but was not statistically significant $(P=\mathrm{NS})$ than that of corresponding vehicletreated WTs (Figure 3a). This may suggest lower baseline $\mathrm{H}_{2} \mathrm{O}_{2}$ results from mCAT activity in mitochondria of TG hearts. $\mathrm{H}_{2} \mathrm{O}_{2}$ abundance in mitochondria from hearts of AZT-treated mCAT TGs remained low, suggesting that the mitochondrial-targeted CAT TG were effectively protected from the oxidative effects of AZT.

\section{Cardiac Aconitase Activity in TGs and WTs}

Decreased aconitase activity (ie, aconitase inactivation) served as a sensitive indicator of oxidative stress. Aconitase activity decreased in heart samples from AZT-treated WTs and from both vehicle or AZT-treated SOD2 ${ }^{+/-} \mathrm{KOs}$ $(P<0.05) \quad$ (Figure 3b). In contrast, aconitase activity remained unchanged in hearts from SOD2-OX and mCAT TGs regardless of treatment compared with vehicle-treated WTs (data not shown). 
a

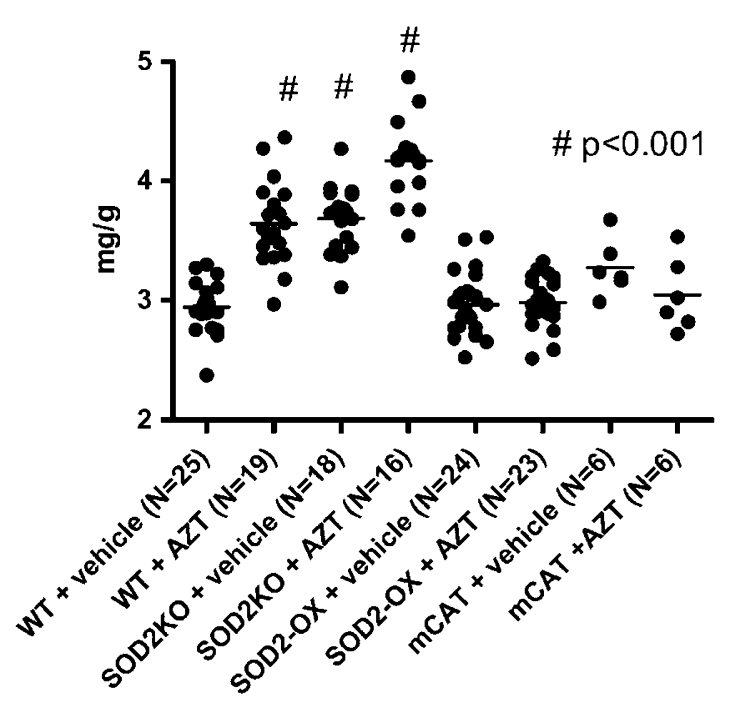

b

LVEDD/bw

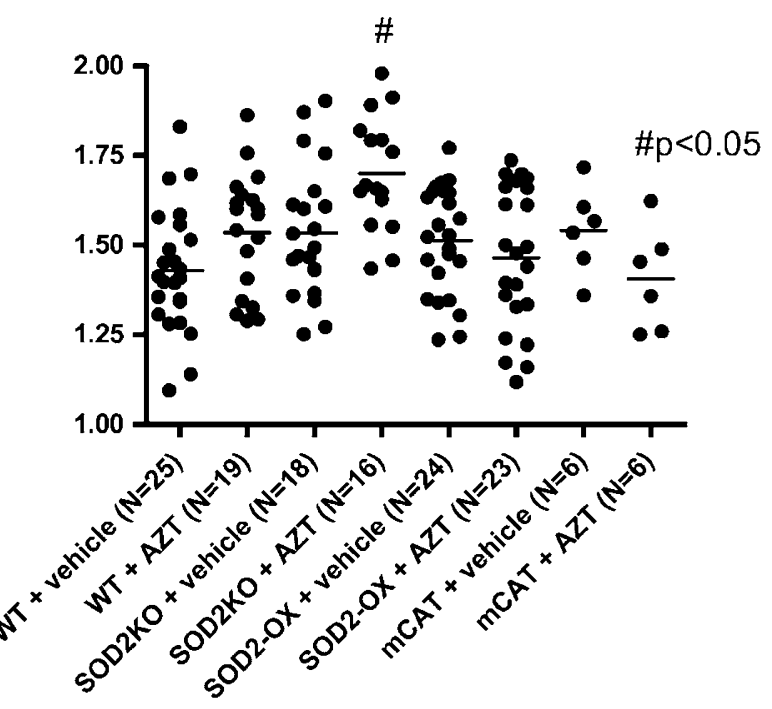

Figure 2 Cardiac functional phenotype in SOD2 ${ }^{+/-}$KO, SOD2-OX and mCAT TGs with and without AZT: SOD2 ${ }^{+/-}$KO, SOD2-OX, mCAT TGs and WT mice were treated with vehicle or with vehicle + AZT through gavage $(0.22 \mathrm{mg}$ per day; 35 days) that was administered in ' 2 by 2 ' factorial protocols. (a) Echocardiographically determined LV mass increased in vehicle-treated SOD2 ${ }^{+/-}$KOs compared with vehicle-treated WTs. AZT treatment increased LV mass in WTs. AZT treatment further increased LV mass in SOD2 ${ }^{+/-}$KOs $\left({ }^{*} P<0.001\right)$. SOD2-OX and mCAT TGs both seemed protected from AZT-induced toxicity. (b) SOD2 ${ }^{+/-}$KO LVEDD was unchanged from LVEDD in WTs. LVEDD was significantly increased by AZT in the SOD2 ${ }^{+/-}$KO $(" P<0.05)$. SOD2-OX and mCAT TGs remained unchanged from WTs regardless of treatment.

a

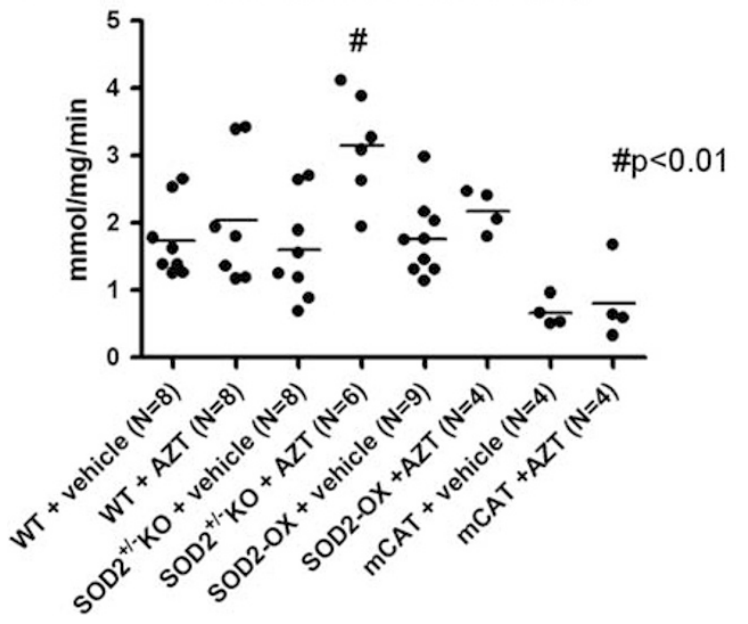

b

Aconitase Activity

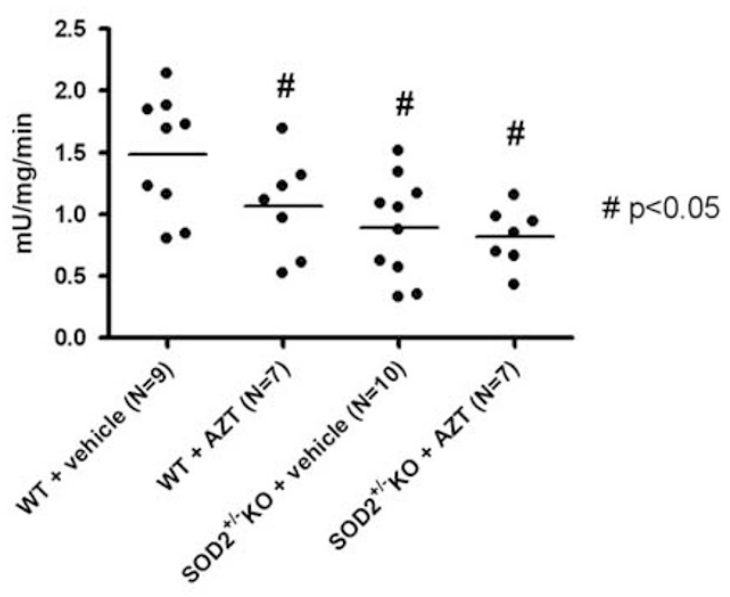

Figure 3 Oxidative phenotype of $\mathrm{CM}$ in SOD2 ${ }^{+/-} \mathrm{KO}$, SOD2-OX and mCAT TGs with and without AZT. (a) Mitochondrial $\mathrm{H}_{2} \mathrm{O}_{2}$ abundance in heart samples from AZT-treated $\mathrm{SOD}^{+/-} \mathrm{KO}$ increased compared with $\mathrm{H}_{2} \mathrm{O}_{2}$ abundance in mitochondrial samples from vehicle-treated SOD ${ }^{+1-} \mathrm{KOs}$ or WT controls $\left({ }^{*} P<0.01\right)$. Mitochondrial $\mathrm{H}_{2} \mathrm{O}_{2}$ abundance remained unchanged in SOD2-OX or decreased in mCAT TGs even after AZT treatment. (b) Decreased cardiac aconitase activity was found in AZT-treated WTs and SOD2 ${ }^{+1-}$ KOs (vehicle or AZT-treated) compared with vehicle-treated WTs $(P<0.05)$.

\section{Histological Changes in WTs, KOs and TGs After AZT Treatment}

Histological changes in $\mathrm{CM}$ are generally non-specific. ${ }^{27}$ However, it was important to determine whether characteristic or unusual changes occurred in this toxic CM model. H\&E-stained sections of hearts from each of the TGs and WTs with and without AZT treatment were analyzed
(Figure 4). AZT disrupted myocardial fibers in WTs. This confirmed microscopic damage from AZT, as seen in other studies. ${ }^{28}$ Myocytolytic changes in vehicle-treated SOD2 ${ }^{+/-}$ KO resembled that of AZT-treated WTs, suggesting a background level of damage in SOD2 ${ }^{+/-} \mathrm{KO}^{29}$ AZT caused myocytolysis (loss and clarification of sarcoplasmic filaments frequently seen in cardiomyopathy $^{7}$ ) in $\mathrm{SOD}^{+/-} \mathrm{KO}$. 
Increased sarcoplasmic granularity (associated with increased density of or increased size of mitochondria) also was seen focally. Microscopic changes, particularly myocytolysis, were
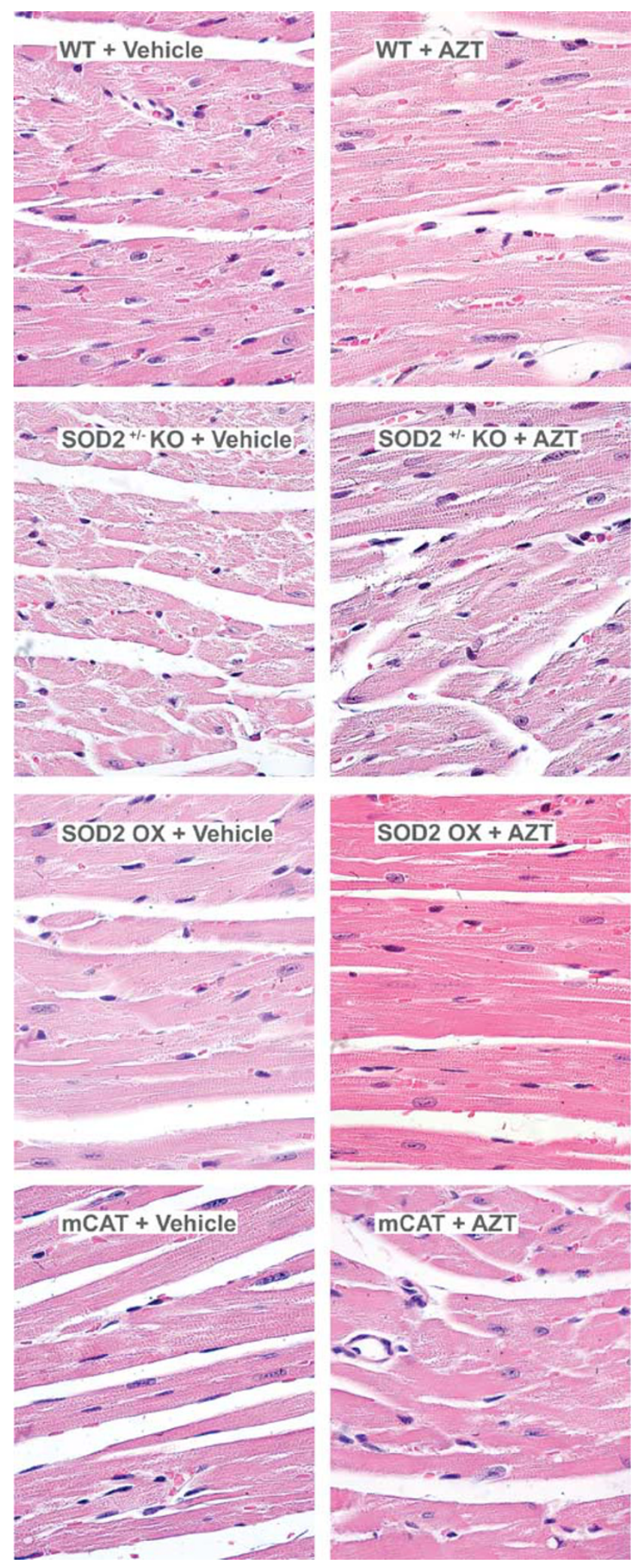

unremarkable in heart samples from SOD2-OX and mCAT TGs (Figure 4). This histopathological finding served as a correlate of the salutary effect of SOD2 or mCAT overexpression and prevention of CM from AZT.

Pathological changes were evaluated comparatively and semiquantitatively. To apply a metric to the microscopic observations, myocyte damage was assessed by blinded reviewers from multiple high-power field views of histology sections for each cohort. ${ }^{30}$ The amount of myocytolysis was scored semiquantitatively on a scale from zero (no myocyte damage) to $4+(>10$ damaged myocytes $)$ at high-power views. Average scores for each cohort are presented (Table 1). Only SOD2 KOs treated with AZT resulted in significant change in number of damaged myocytes from untreated, control WTs.

\section{Ultrastructural Changes in Cardiac Mitochondria}

Heart samples from KOs, TGs and WTs were evaluated for ultrastructural pathological changes. In WTs, AZT caused disruption of mitochondrial cristae (Figure 5). EM features in cardiac mitochondria from vehicle-treated SOD2 ${ }^{+/-} \mathrm{KOs}$ included disruption of cristae (Figure 5). Mitochondrial matrix of cardiac myocytes from AZT-treated SOD2 ${ }^{+/-} \mathrm{KOs}$ revealed worse damage. In contrast, hearts from SOD2-OX and mCAT TGs exhibited mitochondria that resembled those

Table 1 Semi-quantitative analysis of mycocytolysis ${ }^{a}$

\begin{tabular}{llcc}
\hline Mouse & Treatment & Average $( \pm$ s.d.) & $P$-value \\
\hline WT & CMC & $0.4( \pm 0.54)$ & NA \\
WT & AZT & $1.75( \pm 0.5)$ & NS \\
MCAT & CMC & $1.5( \pm 1.05)$ & NS \\
MCAT & AZT & $2.0( \pm 0.0)$ & NS \\
SOD2-OX & CMC & $1.33( \pm 0.52)$ & NS \\
SOD2-OX & AZT & $1.67( \pm 0.52)$ & NS \\
SOD2 KO & CMC & $1.57( \pm 0.79)$ & NS \\
SOD2 KO & AZT & $2.33( \pm 1.15)$ & $P<0.05$
\end{tabular}

${ }^{\mathrm{a}}$ Scored on a scale of $0-4$ (based on number of damaged myocytes/highpower field): 0 myocytes, 0; 1-3 myocytes, 1; 4-6 myocytes, 2; 7-9 myocytes, 3; $10+$ myocytes, 4.

${ }^{\mathrm{b}}$ Non-parametric change from WT/CMC.

NS, non-significant; NA, not applicable.

Figure 4 Structural phenotypes of CM in SOD2 ${ }^{+/-} \mathrm{KO}$, SOD2-OX and mCAT TGs with and without AZT. Representative photomicrographs from ventricular cardiac myocytes of each cohort treated with and without AZT showed disrupted myocardial fibers and myocytolysis in AZT-treated WTs. Pathological changes were exacerbated in SOD2 ${ }^{+/-}$KO. SOD2-OX and mCAT TGs maintained structural integrity of their myocardial fibers even with AZT treatment. (H\&E; original magnification: $\times 600$ ). 

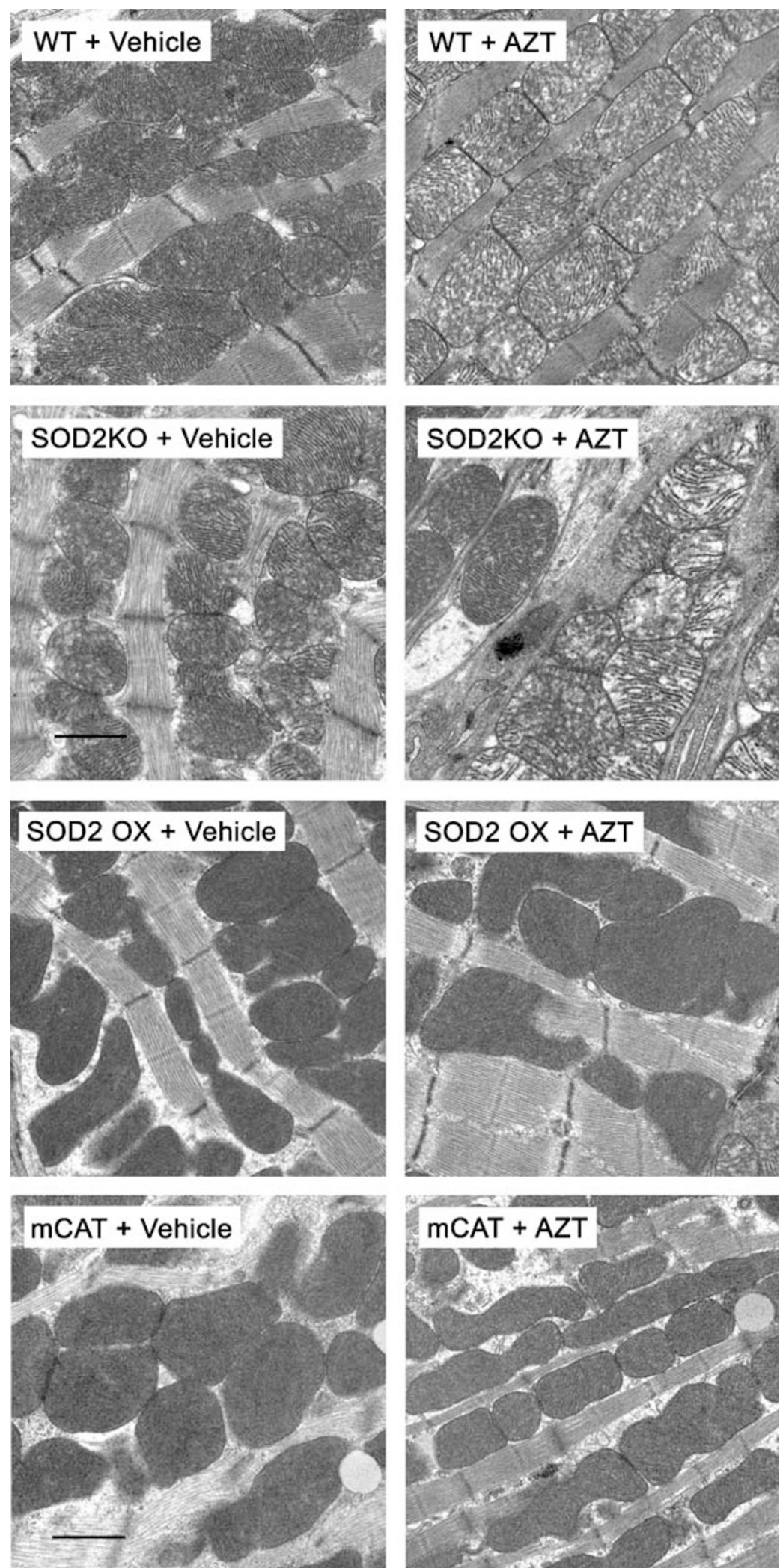

Figure 5 Ultrastructural changes in cardiac mitochondria in SOD2 ${ }^{+/-} \mathrm{KO}$, SOD2-OX and mCAT TGs with and without AZT. Representative TEM profiles of cardiac myocytes from each cohort (with and without AZT) showed cardiac myocytes with disrupted sarcomeres, tubules and mitochondria in vehicletreated SOD2 ${ }^{+/-}$KOs that was worsened by AZT treatment. Changes in cardiac myocytes from SOD2-OX and mCAT TGs (regardless of treatment) were unremarkable and resembled myocytes from vehicle-treated WTs. (original magnification: $\times 23300$ ). 
of vehicle-treated WTs, even after 5 weeks AZT-treatment (Figure 5). Increased abundance of peroxisomes was noted in some cardiac myocyte profiles from mCAT TGs (Figure 5).

\section{DISCUSSION}

Mitochondrial toxic side effects limit therapeutic efficacy and clinical options in HIV/AIDS. AZT mitochondrial toxicity is reported in skeletal muscle ${ }^{31}$ and heart ${ }^{32}$ in vivo and may relate to disrupted mtDNA replication and energetics, but mechanisms remain incompletely understood. Oxidative stress and mtDNA depletion have been the suggested mechanisms, particularly in CM. ${ }^{33-46}$ They may be critical pathogenetically because reactive oxygen species (ROS) are produced abundantly in mitochondria when electron transport chain (ETC) activity is disrupted. ${ }^{47-49}$ ROS are also known to damage ETC complexes cyclically leading to increased ROS (induced by AZT), impaired respiration and further increases in ROS production, etc. ${ }^{50}$

TG models of mitochondrial oxidative stress are useful to study mechanisms of illnesses (reviewed $i^{51}$ ). To define mitochondrial toxic mechanisms of AZT-induced CM, an integrated analysis of cardiac structure, function and mitochondrial oxidative events was accomplished using ' 2 by 2' factorial protocols with proven TG models that enhanced AZT-induced CM and mitochondrial oxidative stress (in the case of SOD2 $2^{+1-} \mathrm{KO}$ ) or protected the heart from it (in the cases of SOD-OX and mCAT). The combination of TGs used in experiments helped define subcellular mechanisms of AZT-induced CM. The proposed mitochondrial mechanisms are highlighted in Figure 6. Data from experiments here indicate oxidative products of dismutation (ie, $\mathrm{H}_{2} \mathrm{O}_{2}$ ) are

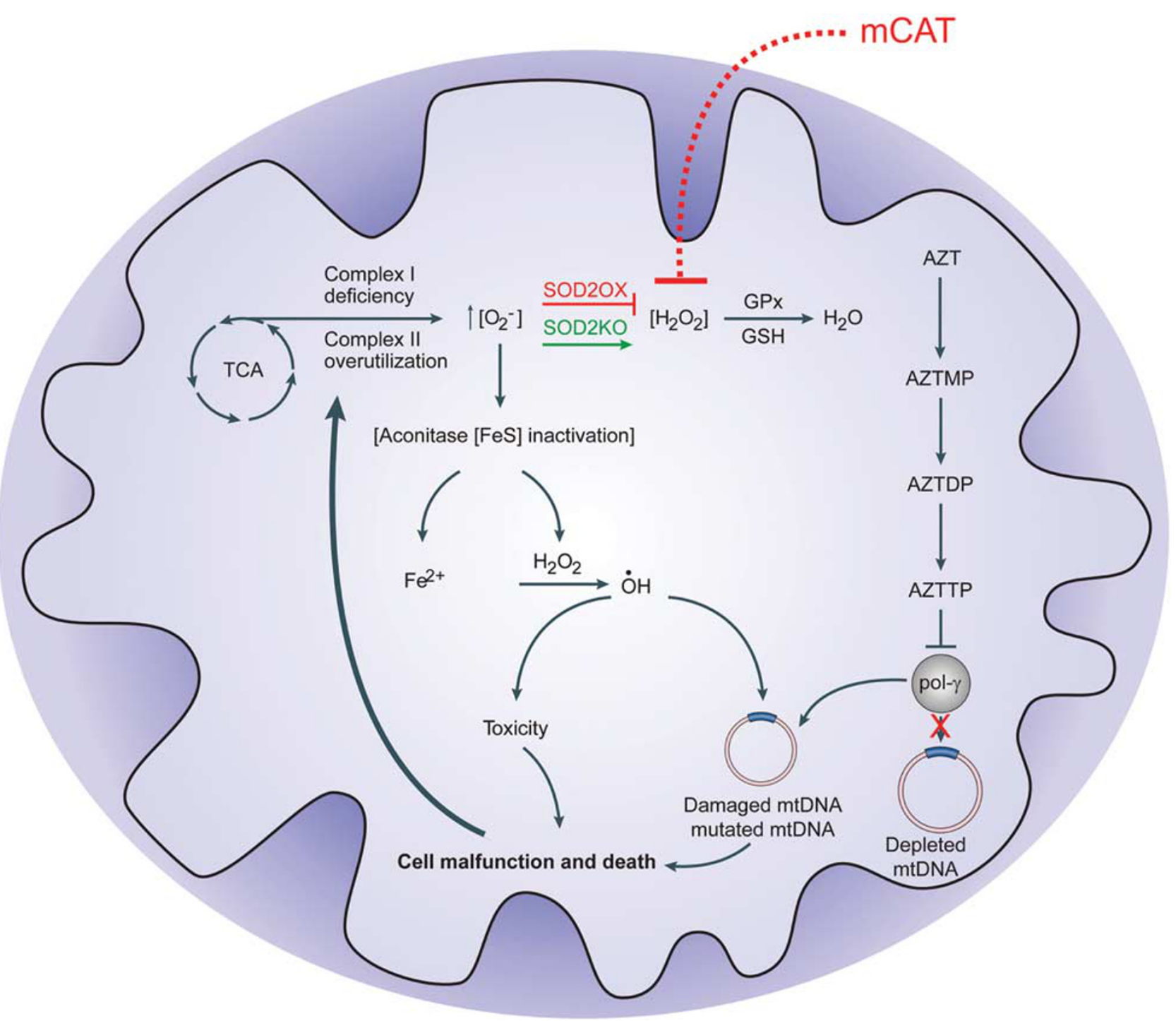

Figure 6 Schematic summary of the proposed mitochondrial mechanisms of AZT toxicity leading to CM. Highlighted are the roles of SOD2 and mCAT overexpression, and/or $\mathrm{KO}$ in regulation of oxidative stress (e $\mathrm{H}_{2} \mathrm{O}_{2}$ production) in the mitochondria that leads to disruption of mitochondrial biogenesis and CM (adapted from 34). 
important factors in mitochondrial CM from NRTIs like AZT.

SOD2 ${ }^{+1-}$ KOs were used to explore mitochondrial oxidative stress in human diseases. ${ }^{19}$ In experiments here, AZT increased cardiac mitochondrial $\mathrm{H}_{2} \mathrm{O}_{2}$ abundance in $\mathrm{SOD} 2^{+/-}$KOs compared with those measurements in either vehicle-treated SOD2 ${ }^{+/-}$KOs or WT controls. To corroborate these findings, myocardial aconitase activity was significantly lower in SOD2 $2^{+/-}$KOs compared with vehicletreated WTs. These results underscore the importance of $\mathrm{H}_{2} \mathrm{O}_{2}$ in the transition to physiological decompensation (ie, $\mathrm{LV}$ dilation) in AZT-induced CM.

Compared with SOD2 ${ }^{+/-}$KOs, SOD2-OX and mCAT TG expression each caused reciprocal events to occur. Biologically, $\mathrm{H}_{2} \mathrm{O}_{2}$ abundance in hearts from vehicle-treated SOD2OX TGs were similar to WTs, and AZT treatment caused slight increase in cardiac mitochondrial $\mathrm{H}_{2} \mathrm{O}_{2}$ abundance just as in AZT-treated WTs. Aconitase activity was unchanged in SOD2-OX TGs compared with WTs and corroborated the findings of mitochondrial $\mathrm{H}_{2} \mathrm{O}_{2}$ abundance. In studies here, aconitase assays were carried out on extracts from LV homogenates. Accordingly, the provenance of aconitase may be from either cytosol or mitochondria from the tissue. Aconitase activity is localized primarily to the mitochondria, ${ }^{52}$ however, some cytoplasmic activity is present. Nonetheless, it is reasonable to think that aconitase activity in the heart homogenate was predominantly from the mitochondria. Together, these data indicated a decreased propensity for or susceptibility to oxidative stress (and CM) in the SOD2OX TGs. SOD2-OX protected cardiac myocytes from histological lytic changes compared with hearts of AZT-treated WTs, in which myocytolysis was a prominent histopathological finding (Table 1).

mCAT protects from oxidative stress in an aging model. ${ }^{22}$ In studies here with mCAT TGs (with or without AZT), LV Mass and LVEDD were both unchanged compared with vehicle-treated WTs. Both mCAT TG cohorts exhibited significantly lower cardiac mitochondrial $\mathrm{H}_{2} \mathrm{O}_{2}$ abundance than corresponding AZT-treated SOD2-OX TGs or WTs. It is reasonable to conclude that overexpression of mCAT prevents accumulation of cardiac mitochondrial $\mathrm{H}_{2} \mathrm{O}_{2}$ and development of $\mathrm{CM}$. It further may be reasonable to expect mCAT TGs to resist forms of CM, in which oxidative stress is central.

The sine qua non in both SOD2-OX and mCAT TGs was prevention of AZT-induced CM. However, the observed pathophysiological events may have resulted from different mechanisms. In the failing myocardium, superoxide inactivates aconitase by oxidizing the $4 \mathrm{Fe} 4 \mathrm{~S}$ cluster making it unstable, thereby resulting in Fe release. ${ }^{53}$ The liberated $\mathrm{Fe}$ then reacts with $\mathrm{H}_{2} \mathrm{O}_{2}$ to produce hydroxyl radical, a reactive species that may be the ultimate causative agent. Some studies have shown that reduction in hydroxyl radical formation can reduce or attenuate myocardial damage. ${ }^{11,54}$ Thus, increasing either SOD or catalase activity in mitochondria should protect against such changes and thereby prevent or attenuate CM. Increased SOD2 or catalase activity here was sufficient to prevent AZT-induced CM. It is reasonable to consider that the prevention of production or accumulation of $\mathrm{O}_{2}^{-}$or $\mathrm{H}_{2} \mathrm{O}_{2}$ intramitochondrially may be an important therapeutic approach. Mitochondrial glutathione peroxidase and peroxiredoxins are antioxidant systems that are also capable of eliminating $\mathrm{H}_{2} \mathrm{O}_{2}$ from cardiac mitochondria.

The relationship between SOD enzyme activity and production of $\mathrm{H}_{2} \mathrm{O}_{2}$ remains somewhat controversial. ${ }^{55,56} \mathrm{~A}$ widely accepted paradigm proposed by Fridovich ${ }^{56}$ states that an increase in enzyme alone is insufficient to yield increased product. The dismutation reaction is dependent stoichiometrically on the substrate concentration (ie, $\mathrm{O}_{2}^{-}$), and transgenic overexpression of the SOD2 does not impact substrate concentration. In support of Fridovich's thesis, results from studies here with SOD2-OX TGs, showed that overexpression of SOD2 did not increase $\mathrm{H}_{2} \mathrm{O}_{2}$ production.

In summary, AZT increased abundance of $\mathrm{H}_{2} \mathrm{O}_{2}$ in cardiac mitochondria and concomitantly decreased myocardial aconitase activity. Pathologically, myocytolysis that occurred along with mitochondrial ultrastructural defects from AZT were amplified in SOD2 ${ }^{+1-}$ KOs. CM was reversed or attenuated in SOD2-OX and mCAT TGs. Through the use of genetically engineered mice, $\mathrm{H}_{2} \mathrm{O}_{2}$ and oxidative stress from AZT were linked mechanistically to mitochondrial CM. It may be reasonable to consider antioxidant therapy for AZT $\mathrm{CM}$ or other forms of mitochondrial $\mathrm{CM}$ on reducing the abundance of $\mathrm{H}_{2} \mathrm{O}_{2}$ in mitochondria.

\section{ACKNOWLEDGEMENTS}

We thank the Department of Pathology Experimental Core Laboratory for their support. The studies were supported by R01 HL059798 and R01 HL079867 to WL.

1. Hammer SM, Saag MS, Schechter M, et al. Treatment for adult HIV infection: 2006 recommendations of the International AIDS SocietyUSA panel. JAMA 2006;296:827-843.

2. Lewis W, Grupp IL, Grupp G, et al. Cardiac dysfunction occurs in the HIV-1 transgenic mouse treated with zidovudine. Lab Invest 2000;80:187-197.

3. Corcuera Pindado MT, Lopez Bravo A, Martinez-Rodriguez R, et al. Histochemical and ultrastructural changes induced by zidovudine in mitochondria of rat cardiac muscle. Eur J Histochem 1994;38:311-318.

4. Ruga E, Bova S, Nussdorfer G, et al. Zidovudine-induced alterations in the heart and vascular smooth muscle of the rat. Cardiovasc Res 2003;60:147-155.

5. Mak IT, Goldfarb MG, Weglicki WB, et al. Cardiac pathologic effects of azidothymidine (AZT) in Mg-deficient mice. Cardiovasc Toxicol 2004;4:169-177.

6. Purevjav E, Nelson DP, Varela JJ, et al. Myocardial Fas ligand expression increases susceptibility to AZT-induced cardiomyopathy. Cardiovasc Toxicol 2007;7:255-263.

7. Lewis W, Dalakas MC. Mitochondrial toxicity of antiviral drugs. Nat Med 1995;1:417-422.

8. McCord JM, Fridovich I. Superoxide dismutase. An enzymic function for erythrocuprein (hemocuprein). J Biol Chem 1969;244:6049-6055.

9. Buckley BJ, Tanswell AK, Freeman BA. Liposome-mediated augmentation of catalase in alveolar type II cells protects against H2O2 injury. J Appl Physiol 1987;63:359-367.

10. Matsushima $S$, Kinugawa $S$, Ide $T$, et al. Overexpression of glutathione peroxidase attenuates myocardial remodeling and preserves diastolic 
function in diabetic heart. Am J Physiol Heart Circ Physiol 2006;291:H2237-H2245.

11. Matsushima $S$, Ide $T$, Yamatgro $M$, et al. Overexpression of mitochondrial peroxiredoxin-3 prevents left ventricular remodeling and failure after myocardial infarction in mice. Circulation 2006;113:1779-1786.

12. Kim H, Lee TH, Park ES, et al. Role of peroxiredoxins in regulating intracellular hydrogen peroxide and hydrogen peroxide-induced apoptosis in thyroid cells. J Biol Chem 2000;275:18266-18270.

13. Reinartz M, Ding Z, Flogel U, et al. Nitrosative stress leads to protein glutathiolation, increased s-nitrosation, and up-regulation of peroxiredoxins in the heart. J Biol Chem 2008;283:17440-17449.

14. Halliwell B, Gutteridge JM. The importance of free radicals and catalytic metal ions in human diseases. Mol Aspects Med 1985;8:89-193.

15. Shioji K, Kishimoto C, Nakamura $\mathrm{H}$, et al. Overexpression of thioredoxin-1 in transgenic mice attenuates adriamycin-induced cardiotoxicity. Circulation 2002;106:1403-1409.

16. Schrader M, Fahimi HD. Peroxisomes and oxidative stress. Biochim Biophys Acta 2006;1763:1755-1766.

17. Oshino $\mathrm{N}$, Chance $\mathrm{B}$, Sies $\mathrm{H}$, et al. The role of $\mathrm{H}_{2} \mathrm{O}_{2}$ generation in perfused rat liver and the reaction of catalase compound I and hydrogen donors. Arch Biochem Biophys 1973;154:117-131.

18. Boveris A, Oshino N, Chance B. The cellular production of hydrogen peroxide. Biochem J 1972;128:617-630.

19. Melov S, Schneider JA, Day BJ, et al. A novel neurological phenotype in mice lacking mitochondrial manganese superoxide dismutase. Nat Genet 1998;18:159-163.

20. Chen Z, Siu B, Ho YS, et al. Overexpression of MnSOD protects against myocardial ischemia/reperfusion injury in transgenic mice. J Mol Cell Cardiol 1998;30:2281-2289.

21. Ho Y S, Magnenat JL, Gargano $M$, et al. The nature of antioxidant defense mechanisms: a lesson from transgenic studies. Environ Health Perspect 1998;106:1219-1228.

22. Schriner SE, Linford NJ, Martin GM, et al. Extension of murine life span by overexpression of catalase targeted to mitochondria. Science 2005;308:1909-1911.

23. Patel M, Day BJ. Metalloporphyrin class of therapeutic catalytic antioxidants. Trends Pharmacol Sci 1999;20:359-364.

24. Patel M, Day BJ, Crapo JD, et al. Requirement for superoxide in excitotoxic cell death. Neuron 1996;16:345-355.

25. Lewis W, Day BJ, Kohler JJ, et al. Decreased mtDNA, oxidative stress, cardiomyopathy, and death from transgenic cardiac targeted human mutant polymerase gamma. Lab Invest 2007;87:326-335.

26. Lewis W, Haase CP, Raidel SM, et al. Combined antiretroviral therapy causes cardiomyopathy and elevates plasma lactate in transgenic AIDS mice. Lab Invest 2001;81:1527-1536.

27. Gallo P, d'Amati G, In: Silver MD, Gotlieb Al, Schoen FJ (eds). Cardiovascular Pathology. Churchill Livingstone: Philadelphia, USA, 2001, pp 285-325.

28. Lewis W, Papoian T, Gonzalez B, et al. Mitochondrial ultrastructural and molecular changes induced by zidovudine in rat hearts. Lab Invest 1991;65:228-236.

29. Williams MD, Van Remmen H, Conrad CC, et al. Increased oxidative damage is correlated to altered mitochondrial function in heterozygous manganese superoxide dismutase knockout mice. J Biol Chem 1998;273:28510-28515.

30. Sutliff RL, Haase C, Russ R, et al. Cocaine increases mortality and cardiac mass in a murine transgenic model of acquired immune deficiency syndrome. Lab Invest 2003;83:983-989.

31. Dalakas MC, Illa I, Pezeshkpour GH, et al. Mitochondrial myopathy caused by long-term zidovudine therapy. N Engl J Med 1990;322:1098-1105.

32. Lewis W, Currie PF, In: Fuster V, O'Rourke RA, Walsh RA, Poole-Wilson P (eds). Hurst's The Heart. McGraw Hill Medical: New York, USA, 2008, pp 2118-2131.

33. Szabados E, Fischer GM, Toth $\mathrm{K}$, et al. Role of reactive oxygen species and poly-ADP-ribose polymerase in the development of AZT-induced cardiomyopathy in rat. Free Radic Biol Med 1999;26:309-317.

34. Lewis W, Day BJ, Copeland WC. Mitochondrial toxicity of nrti antiviral drugs: an integrated cellular perspective. Nat Rev Drug Discov 2003;2:812-822.
35. Bialkowska A, Bialkowski K, Gerschenson M, et al. Oxidative DNA damage in fetal tissues after transplacental exposure to $3^{\prime}$-azido- $3^{\prime}$ deoxythymidine (AZT). Carcinogenesis 2000;21:1059-1062.

36. Caron M, Auclairt M, Vissian A, et al. Contribution of mitochondrial dysfunction and oxidative stress to cellular premature senescence induced by antiretroviral thymidine analogues. Antivir Ther 2008;13:27-38

37. de la Asuncion JG, Del Olmo ML, Gomez-Cambronero LG, et al. AZT induces oxidative damage to cardiac mitochondria: protective effect of vitamins C and E. Life Sci 2004;76:47-56.

38. de la Asuncion JG, del Olmo ML, Sastre J, et al. AZT treatment induces molecular and ultrastructural oxidative damage to muscle mitochondria. Prevention by antioxidant vitamins. J Clin Invest 1998;102:4-9.

39. Deveaud C, Beauvoit B, Reynaud A, et al. Site-specific reduction of oxidative and lipid metabolism in adipose tissue of $3^{\prime}$-azido-3'deoxythymidine-treated rats. Antimicrob Agents Chemother 2007;51:583-590.

40. Dunge A, Chakraborti AK, Singh S. Mechanistic explanation to the variable degradation behaviour of stavudine and zidovudine under hydrolytic, oxidative and photolytic conditions. J Pharm Biomed Anal 2004;35:965-970.

41. Ferraresi $R$, Troiano $L$, Roat $E$, et al. Protective effect of acetyl-Lcarnitine against oxidative stress induced by antiretroviral drugs. FEBS Lett 2006;580:6612-6616.

42. Iwamoto $T$, Hiraku Y, Oikawa $S$, et al. Oxidative DNA damage induced by photodegradation products of $3\left({ }^{\prime}\right)$-azido-3(')-deoxythymidine. Arch Biochem Biophys 2003;416:155-163.

43. Lagathu C, Eustace B, Prot M, et al. Some HIV antiretrovirals increase oxidative stress and alter chemokine, cytokine or adiponectin production in human adipocytes and macrophages. Antivir Ther 2007;12:489-500.

44. Mondal D, Pradhan L, Ali M, et al. HAART drugs induce oxidative stress in human endothelial cells and increase endothelial recruitment of mononuclear cells: exacerbation by inflammatory cytokines and amelioration by antioxidants. Cardiovasc Toxicol 2004;4:287-302

45. Romero-Alvira D, Roche E. The keys of oxidative stress in acquired immune deficiency syndrome apoptosis. Med Hypotheses 1998;51:169-173.

46. Benjamin IJ, Schneider MD. Learning from failure: congestive heart failure in the postgenomic age. J Clin Invest 2005;115:495-499.

47. Chance B, Sies $\mathrm{H}$, Boveris A. Hydroperoxide metabolism in mammalian organs. Physiol Rev 1979;59:527-605.

48. Lewis W, Gonzalez B, Chomyn A, et al. Zidovudine induces molecular, biochemical, and ultrastructural changes in rat skeletal muscle mitochondria. J Clin Invest 1992;89:1354-1360.

49. Rosca MG, Vazquez EJ, Kerner J, et al. Cardiac mitochondria in heart failure: decrease in respirasomes and oxidative phosphorylation. Cardiovasc Res 2008;80:30-39.

50. Korge $\mathrm{P}$, Ping $\mathrm{P}$, Weiss JN. Reactive oxygen species production in energized cardiac mitochondria during hypoxia/reoxygenation: modulation by nitric oxide. Circ Res 2008;103:873-880.

51. Wallace DC. A mitochondrial paradigm of metabolic and degenerative diseases, aging, and cancer: a dawn for evolutionary medicine. Annu Rev Genet 2005;39:359-407.

52. Mason KE, Stofan DA. Endotoxin challenge reduces aconitase activity in myocardial tissue. Arch Biochem Biophys 2008;469:151-156.

53. Ide T, Tsutsui $H$, Hayashidani $\mathrm{S}$, et al. Mitochondrial DNA damage and dysfunction associated with oxidative stress in failing hearts after myocardial infarction. Circ Res 2001;88:529-535.

54. Hiranandani N, Bupha-Intr T, Janssen PM. SERCA overexpression reduces hydroxyl radical injury in murine myocardium. Am J Physiol Heart Circ Physiol 2006;291:H3130-H3135.

55. Buettner $\mathrm{GR}, \mathrm{Ng} \mathrm{CF}$, Wang $\mathrm{M}$, et al. A new paradigm: manganese superoxide dismutase influences the production of $\mathrm{H} 2 \mathrm{O} 2$ in cells and thereby their biological state. Free Radic Biol Med 2006;41:1338-1350.

56. Fridovich I. Mitochondria: are they the seat of senescence? Aging Cell 2004;3:13-16. 